\title{
LOS EFECTOS DE LA RECONCENTRACIÓN (1896-98) EN LA SOCIEDAD CUBANA. UN ESTUDIO DE CASO: GÜIRA DE MELENA
}

POR

\author{
FRANCISCO PÉREZ GUZMÁN
}

Instituto de Historia de Cuba

\begin{abstract}
La Reconcentración en Güira de Melena es un estudio de caso que aborda las consecuencias sociales, económicas e históricas. En el trabajo se enfatiza la magnitud de las defunciones en la población civil y las causas de muertes; fundamentado en fuentes primarias se exponen las tendencias registradas que evidencian un sangramiento demográfico.
\end{abstract}

Este trabajo se enmarca en el proyecto de investigación «La Reconcentración» que actualmente realizamos. Por tanto, es un estudio de caso cuyas particularidades se insertan dentro de la generalidad de la Reconcentración (1896-98) y contribuye a revelar la magnitud que como trauma histórico social repercutió en la población cubana.

Uno de los objetivos planteados es conocer cómo incidió la Reconcentración en la elevación de la mortandad en la población y aproximarnos a sus efectos sociales. Para cumplimentarlo revisamos unas 3.400 actas de defunciones. Esta lectura posibilitó saber cuáles fueron las enfermedades que influyeron en las causas de muertes como consecuencia de la Reconcentración.

En la investigación trabajamos con las variantes siguientes: año, día, edad, sexo, raza, causa de muerte, estado civil y lugar de residencia. A su vez, los fallecidos los clasificamos por grupos de edades según la metodología aplicada por los epidemiólogos en la elaboración de sus trabajos científicos. La selección de Güira de Melena como estudio de caso se fundamenta, en primer lugar, por disponer el autor de fuentes suficientes. 
El éxito de la invasión mambisa al Occidente de Cuba determinó un cambio sustancial en la concepción de la guerra que hasta esa fecha (enero de 1896) había efectuado Madrid. El conservador Cánovas del Castillo, al frente del Consejo de Ministros, en un esfuerzo por recuperar la credibilidad del dominio español en la Isla ante los estados europeos y de Estados Unidos de América y apaciguar la inquietud de los sectores políticos de la sociedad española, elaboró un conjunto de medidas destinadas a neutralizar el auge alcanzado por la revolución.

Con ese propósito nombró a Valeriano Weyler y Nicoláus como nuevo Capitán General de la Isla ${ }^{1}$. Se enviaron al escenario bélico refuerzos en soldados y oficiales experimentados, cientos de piezas de artillería, embarcaciones para el patrullaje de las costas para evitar el desembarco de expediciones. Además, gestionó empréstitos imprescindibles para cubrir los gastos de guerra que se incrementaban de forma vertical.

Valeriano Weyler definió la nueva política de guerra como guerra con la guerra.

Dentro del conjunto de medidas que se implementaría para materializar los objetivos estratégicos de pacificar la Isla, la Reconcentración de campesinos y residentes rurales era considerada como una de las más importantes. Con ella aspiraban, en primer lugar, cortar todos los suministros de alimentos a los insurrectos; así como reducir al mínimo sus potencialidades en el arma de caballería al no poder reemplazar las pérdidas de caballos debido a los combates.

A su vez, la Reconcentración privaría a los insurrectos de informaciones militares que le facilitaban los simpatizantes de la causa independentista. Por otra parte, el traslado de miles de jóvenes a los centros urbanos evitaría el reclutamiento de ellos.

La intensificación de los trabajos defensivos de ciudades y pueblos frustraría gran parte de los ataques mambises con pretensiones

\footnotetext{
ABREVIATURA UTILIZADA:

ANC, Archivo Nacional de Cuba (La Habana).

1 Valeriano Weyler, marqués de Tenerife, había participado con anterioridad en la Guerra de Restauración en Santo Domingo. En Cuba combatió durante la Guerra de los Diez Años a las órdenes del tristemente célebre conde de Valmaseda. Recordado por su crueldad y aplicar en Oriente el método de la Reconcentración.
} 
de abastecerse de artículos de primera necesidad, más la prohibición de sacar mercancías de los centros urbanos con destino a zonas rurales y la Reconcentración, eran medidas encaminadas a rendir por hambre, enfermedades y desmoralización, ante la falta de perspectiva de triunfo, al Ejército Libertador.

Güira de Melena, municipio habanero situado en el suroeste de la provincia, se caracterizaba en la década del noventa del siglo XIX por una elevada y sostenida producción agrícola. Era uno de los principales proveedores de viandas a la capital, como plátanos, papas, yuca, malanga y hortalizas. También se cultivaba el tabaco y la caña de azúcar.

La producción agrícola se originaba en la mediana y pequeña propiedad, aunque esta última era la predominante. Al respecto, la referencia más cercana a los días de guerra que pudiera darnos una información ilustrativa se remonta a 1884. En este año el censo municipal expresaba que las fincas rústicas alcanzaban la cifra de 310 y las azucareras eran 15.

Muy pronto los campesinos y obreros agrícolas experimentaron los efectos de la guerra. El incremento de las actividades bélicas y de las tropas en operaciones, incidieron en la economía y seguridad campesina. Los cubanos obligados por la necesidad se apoderaban del ganado vacuno, porcino, caballar y de viandas y maíz. Por otra parte, cobraban impuestos, quemaban cañaverales y destruían vegas de tabaco con el propósito estratégico de golpear los fundamentos de la base económica de la administración colonial.

Los españoles también se nutrían de los productos agrícolas y de animales. Y emprendieron una represalia feroz contra todos aquellos que sospechaban ser colaboradores de los insurrectos. Sus fincas eran destruidas y las casas incendiadas.

La situación de inseguridad para la población rural motivó que algunos campesinos con sus familias se trasladaran al pueblo. Ese mismo éxodo emprendieron decenas de jornaleros al quedar sin empleo.

No obstante, este proceso inicial emigratorio se caracterizó por ser voluntario y muchos de ellos se instalaron en casas confortables en pueblos y ciudades. Otros marcharon a La Habana y algunos al 
extranjero. Los más pobres trataron de hallar la sobreviviencia en la búsqueda de cualquier trabajo.

Aunque la Reconcentración se instauró el 16 de febrero de 1896 para Sancti Spiritus, Camagüey y Santiago de Cuba, no fue hasta el 21 de octubre de ese año que se aplicó de forma sistemática. La misma se inició por Pinar del Río, después se extendió a las provincias de Matanzas, La Habana y Santa Clara. En su primer artículo ordenaba:

\footnotetext{
"Todos los habitantes en los campos o fuera de la línea de fortificación de los poblados, se reconcentrarán en el término de ocho días en los pueblos ocupados por las tropas. Será considerado rebelde o juzgado como tal, todo individuo que transcurrido ese plazo se encuentre en despoblado."
}

Como complemento —orientado a suprimir los abastecimientos a los mambises - se prohibía en lo absoluto «la extracción de víveres de los poblados y la conducción de uno u otro por mar o tierra, sin permiso de la autoridad militar del punto de partida.» $\mathrm{Y}$ se advertía que a los infractores se juzgaría y penaría como auxiliares de los insurrectos. Además, los propietarios de reses estaban obligados a conducirlas a los pueblos o sus inmediaciones ${ }^{2}$.

A principios de 1897 las familias de Güira de Melena que residían en los barrios rurales como Sabanacán, Melena y Tumbadero, fueron trasladadas por las tropas del ejército y guerrillas, hasta el pueblo. En carretas y carretones depositaron sus pertenencias. También llevaban sus animales de crianza. En el pueblo fueron ubicadas en barracones que habían sido construidos. Pero con el decursar de los meses las que llegaban se instalaban en edificios abandonados y en chozas de tabla de palma, yegua y guano edificadas por ellos mismos. Incluso vivían en lugares públicos a la intemperie.

Cómo sobrevivir fue el gran problema que afrontaron los reconcentrados, pues aunque todos tenían esa condición, entre ellos existían diferencias en cuanto a poder económico, status social y nivel cultural. Para los que llegaron a Güira después de junio de 1896 la situación se tornó más difícil. Las tabaquerías estaban saturadas de

\footnotetext{
2 Aunque en el Bando se subrayaba que era para la provincia de Pinar del Río, más tarde se extendió a La Habana y Matanzas. Con anterioridad, el 16 de febrero de ese año, Weyler publicó un bando de reconcentración que afectaba a Sancti Spíritus, Puerto Príncipe y Santiago de Cuba. Pero el mismo no llegó a aplicarse con rigurosidad hasta finales de 1896.
}

R. I., $1998, \mathrm{n}^{\circ} 212$ 
trabajadores. En los comercios no habían plazas. Los trabajos domésticos escaseaban. Y el empleo de lavandera era casi imposible. Casi la única opción consistía en trabajar en obras públicas para construir carreteras, levantar terraplenes, edificar fortificaciones y en las zonas de cultivos.

Esa situación obligaba a muchos reconcentrados al forrajeo en las fincas abandonadas como una manera de ganar algún dinero al vender viandas, frutas y cañas de azúcar para hacer melao ${ }^{3}$.

\section{III}

La incidencia de la Reconcentración en cuanto a la elevación de las cifras de muertes anuales queda demostrada al revisar su comportamiento en los años de 1897 y 1898, considerados los más dramáticos. En 1893, un año normal ${ }^{4}$, los fallecimientos alcanzaron la cifra de 286 en Güira de Melena. Incluso en el segundo semestre de 1896, en plena guerra, fue de 182 , es decir, 17 fallecidos más que igual semestre de 1893. Sin embargo, en 1897 el número de muertes se disparó hasta 1.444 para descender ligeramente en 1898 a 1.380. $\mathrm{Al}$ respecto es de significar que en ese incremento vertical no influyó de forma determinante las muertes de personas pertenecientes al Ejército de Operaciones en Cuba (español) y al Ejército Libertador. En 1987 se realizaron cinco actas de defunciones de personas muertas en acciones de guerra y en 1898 fue de siete. Y los casos de fiebre amarilla - todos de españoles - puede considerarse insignificante.

En 1893 las primeras diez causas de muertes para la población de Güira de Melena fue la siguiente:

\begin{tabular}{|c|l|c|}
\hline LUGAR & ENFERMEDADES & FALLECIDOS \\
\hline 1 & Enteritis y gastroenteritis & 45 \\
2 & Tuberculosis & 39 \\
3 & Tétanos & 32 \\
4 & Neumonía y Bronconeumonía & 25
\end{tabular}

3 Ramiro Guerra, en su obra testimonial Por la vereda del pasado, relata de forma detallada los modos de los reconcentrados para buscarse la vida.

4 La selección del año 1893 se fundamenta en la disponibilidad de información. Pues en el Registro Civil de Güira de Melena los libros de los años de 1894 y 1895 no se conservan. 


\begin{tabular}{|c|l|c|}
\hline LUGAR & ENFERMEDADES & FALLECIDOS \\
\hline 5 & Del corazón y vasos sanguíneos & 23 \\
6 & Meningitis y meningo encefalitis & 21 \\
7 & Nutricionales y carenciales & 14 \\
8 & Paludismo & 13 \\
9 & Cerebrovasculares & 12 \\
10 & Anomalías congénitas & 11 \\
\hline
\end{tabular}

Fuente: Actas de defunciones del Registro Civil de Güira de Melena. Tomos 11 y 12 .

Es de observar que entre las diez primeras causas de muertes, las enfermedades infecciosas ocupan los lugares 1, 2, 3, 4, 6 y 8; las crónicas el 5 y 9, y las nutricionales el 7.

Como podrá apreciarse, las primeras cuatro causas obedecen a enfermedades transmisibles o infecto contagiosas. El hecho es muy importante porque esas enfermedades están directamente relacionadas con serios problemas higiénicos-sanitarios como la contaminación de aguas y alimentos, mala disposición de residuales líquidos finales (excretos) y sólidos (basuras). Esto nos revela una deficiente infraestructura sanitaria que se agudizará durante la guerra. Esta situación repercutirá de forma nefasta en la población urbana y, en particular, en los cientos de miles de reconcentrados hacinados en locales inapropiados y carentes de recursos para cubrir sus gastos de asistencia médica y de medicinas.

Por otra parte, es interesante el séptimo lugar de causa de muerte ocupado por enfermedades relacionadas directamente con la nutrición o carenciales. Pues los catorce fallecidos que representa el $4,72 \%$ del total, ilustrará no sólo cómo repercutió la Reconcentración en la elevación del número de muertes por esa causa, sino también, en la aparición de enfermedades como la hidroemia, anemia, diateris escorbútica, escorbuto, inanición y acceso pernicioso, que antes se desconocían o eran casos muy raros. Esto lo demuestra que las defunciones realizadas en 1893 por nutrición todas fueron por raquitismo y en su inmensa mayoría en niños menores de cinco años.

El año de 1897 es harto elocuente para entender en toda su magnitud la incidencia de la Reconcentración en la elevación y causas de muertes de la población güireña. En cuanto al comportamiento del incremento, el mismo fue así: 


\begin{tabular}{|lclc|}
\hline MES & FALLECIDOS & MES & FALLECIDOS \\
\hline Enero & 46 & Julio & 147 \\
Febrero & 60 & Agosto & 153 \\
Marzo & 102 & Septiembre & 144 \\
Abril & 116 & Octubre & 148 \\
Mayo & 120 & Noviembre & 156 \\
Junio & 117 & Diciembre & 135 \\
& & Total: & $\mathbf{1 . 4 4 4}$ \\
\hline
\end{tabular}

Fuente: Actas de defunciones del Registro Civil de Güira de Melena. Tomos 11 y 12.

Como referencia comparativa, en 1893 los meses más altos en defunciones fueron julio, agosto y diciembre, con treinta cada uno. Y el mes de menor número fue febrero, con 16.

Aún en el segundo semestre de 1896, a pesar de la situación de guerra y de haberse decretado el bando de Reconcentración en octubre, el número de muertes tuvo un registro muy similar. Pues el comportamiento fue el siguiente:

\begin{tabular}{lc}
\hline MES & FALLECIDOS \\
\hline Julio & 44 \\
Agosto & 35 \\
Septiembre & 21 \\
Octubre & 20 \\
Noviembre & 22 \\
Diciembre & 40 \\
Total & $\mathbf{1 8 2}$ \\
\hline
\end{tabular}

Fuentes: Actas de defunciones del Registro Civil de Güira de Melena. Tomo 9.

Las muertes por meses confirma que las incidencias de la Reconcentración en los decesos se materializaba con el decursar del tiempo y se acentuaba en la temporada de lluvia. Pues fue a partir de marzo de 1897 - aún predominaba la temporada de seca- en que el número de muertes ascendió en flecha. En ese año las principales enfermedades fueron las siguientes: 
PRIMERAS DIEZ CAUSAS DE MUERTES

PARA TODAS LAS EDADES

\begin{tabular}{|c|l|c|}
\hline LUGAR & ENFERMEDADES & FALLECIDOS \\
\hline 1 & Enteritis y gastroenteritis & 245 \\
2 & Tuberculosis & 183 \\
3 & Paludismo & 170 \\
4 & Viruela & 166 \\
5 & Nutricionales y/o carenciales & 107 \\
6 & Fiebre Tifoidea & 71 \\
7 & Del corazón y vasos sanguíneos & 58 \\
8 & Disentería & 42 \\
9 & Cerebrovasculares & 41 \\
10 & Neumonía y Bronconeumonía & 37 \\
\hline
\end{tabular}

Fuentes: Actas de defunciones del Registro Civil de Güira de Melena. Tomos 11 y 12.

Lo más significativo del año 1897 es la inclusión en los lugares sexto y octavo de la fiebre tifoidea y disentería, enfermedades de transmisión digestiva y que con anterioridad se hallaban relegadas. Su incremento, al igual que otras como la enteritis y gastroenteritis (entéricas) radicaba en el empeoramiento de la higiene personal y ambiental. En tal sentido desempeñó un papel influyente el continuo arribo a Güira de familias reconcentradas procedentes de la provincia de Pinar del Río, y de pueblos limítrofes como La Salud, Quivicán, San Antonio de los Baños y Batabanó. Este flujo emigratorio interno fue constante, lo que originó el hacinamiento infernal de esa población.

En el contexto anterior hallamos la respuesta del porqué se elevaron los casos de tuberculosis - considerada epidémica antes de la Reconcentración- y viruela. Pues ambas son de transmisión respiratoria. Claro está que en el caso de la tuberculosis la deficiente nutrición es un factor determinante en el número de muertes registradas.

El tercer lugar ocupado por el paludismo como causa de muerte está supeditado a la proliferación del mosquito Anópheles Albimanus. Recordemos que las poblaciones se hallaban rodeadas de fosos para dificultar los ataques insurrectos. Más las aguas vertidas por la población convirtieron a las zonas urbanas en gigantescos criaderos de ese mosquito y, también, del Aedes Aegyti. Este último transmisor de la fiebre amarilla. Enfermedad que en Güira de Melena no ad- 
quiere importancia como causa de muerte principal, pues en el segundo semestre de 1896 se registraron dos casos, en 1897 cuatro y dos en 1898. Todos eran militares españoles.

La ayuda del gobierno central a los municipios para solucionar las dificultades con las viviendas, higiene y alimentos era muy ínfima. Los ayuntamientos municipales tuvieron que cargar con todo el peso de la problemática y muy poco pudieron hacer porque los ingresos por el concepto de contribución se redujeron a consecuencia de la guerra. Parte del dinero recaudado se destinaba a la construcción de fuertes, alquileres de casas para la oficialidad del Ejército, suministro de materiales diversos a las representaciones de las Brigadas o media brigada en la localidad, atenciones a la cárcel, al cuerpo de bomberos, alumbrado público y los salarios de la burocracia municipal.

La disponibilidad de recursos monetarios y la disposición de las autoridades municipales para auxiliar a los reconcentrados condicionó una desigualdad entre las ciudades y pueblos en relación con la sobrevivencia.

En realidad fueron las organizaciones sociales, religiosas y la voluntad popular la que más contribuyó a aliviar las calamidades sufridas por los reconcentrados y los sectores más empobrecidos de la población.

Un cambio notable acerca de la situación existente comenzó a experimentarse cuando el gobierno de Práxedes Mateo Sagasta se vio obligado a relevar a Valeriano Weyler de la Capitanía General de la Isla. Múltiples factores incidieron en esa sustitución. Entre estos pueden mencionarse: el fracaso de la pacificación de la Isla y el costo político que significaba para España la Reconcentración. Ésta era muy censurada por los estados europeos y, en especial, por el Gobierno de Estados Unidos de América, que la había incluido como un elemento estratégico de su política exterior para legitimar su posible intervención en la guerra de Cuba.

Una de las primeras medidas adoptada por Ramón Blanco y Erenas, como nuevo capitán general, gobernador de la Isla y general en jefe del Ejército de Operaciones, fue la de atenuar la repercusión negativa de la Reconcentración. El 13 de noviembre de 1897, es decir, a unas dos semanas de asumir su cargo, el marqués de Peña Plata decretó el bando por el cual se creaban las Juntas Protectoras, a nivel de ciudades y pueblos, cuya tarea principal consistiría en socorrer a los reconcentrados. 
Las Juntas Protectoras, en lo fundamental, quedarían integradas por personas representativas de la localidad como doctores en medicina, sacerdotes, comerciantes y políticos autonomistas. La directiva casi siempre la conformaban alrededor de seis personas. Las mismas organizarían actividades para recaudar fondos destinados a la compra de alimentos, medicinas y controlarían su distribución. Velarían por el funcionamiento de la asistencia médica y el saneamiento ambiental. Y recepcionarían y distribuirían la ayuda proveniente de Estados Unidos.

En ese mismo mes, el día 23, el gobierno concedió un crédito de 100.000 pesos plata con cargo al presupuesto de guerra. Suma que si bien no era significativa para remediar las calamidades de la población reconcentrada, al menos era muy superior a las aprobadas bajo el mando de Weyler.

El gobierno autonómico instalado en la Isla, el 1 de enero de 1898, comenzó a realizar una intensa labor destinada a normalizar la situación de los reconcentrados. Uno de los primeros acuerdos del Consejo de Secretarios fue la de iniciar una amplia investigación para obtener informaciones reales sobre el estado y necesidades de los reconcentrados. Cómo se comportaba la distribución de la ayuda - se registraron muchísimos casos de corrupción- y conocer las potencialidades de la fuerza de trabajo agrícola para reanudar las faenas productivas.

Con ese marcado fin, los Secretarios de Agricultura y Comercio y de Instrucción Pública recibieron orientaciones para que prepararan un conjunto de medidas en las cuales se incluirían la formación de colonias agrícolas. Se facilitaría terrenos, aperos de labranza, semillas y otros útiles. Además, el gobierno junto con las autoridades militares garantizaron la seguridad de ellos. No sólo de posibles ataques de los mambises, sino también, de los extremismos de las tropas en operaciones.

El 15 de febrero de 1898, el Consejo de Secretarios acordaba «Autorizar en las cuatro provincias occidentales de la Isla y a petición de los particulares, el establecimiento de colonias agrícolas y campos de cultivos, determinando de este modo el regreso a las propiedades rurales y a la reproducción del trabajo en los campos con

R. I., $1998, \mathrm{n}^{\circ} 212$ 
las precauciones que el Gobierno determinará para la mayor seguridad de los mismos» ${ }^{5}$.

Esta determinación no es consecuencia de un aceleramiento de la extinción de la lucha armada en Occidente. Al examinar las acciones bélicas se observa que la misma registra una tendencia sostenida en cuanto a pequeños encuentros, ataques a poblaciones y al transporte militar por vía férrea. El propio texto del acuerdo referido a la seguridad confirma, en parte, la situación de guerra existente.

En realidad, la medida a lo que aspiraba era aliviar la crisis alimentaria, descongestionar a los pueblos y ciudades saturados por una población que la Reconcentración había convertido en indigente y condenado a una muerte a mediano o largo plazo. Además sus condiciones de vida infrahumana lo hacían ser agentes transmisores de enfermedades infestocontagiosas, delincuencia, prostitución y mendicidad.

Los autonomistas conscientes de la necesidad de extirpar las secuelas sociales de la Reconcentración extendieron al Secretario de Obras Públicas la recomendación de emplear en esa dependencia a todos los que pudieran realizar ese trabajo.

A su vez incrementaron el número de las llamadas cocinas económicas o populares, que con fondos del Estado y donaciones mitigaban el hambre entre los niños y las familias más desamparadas. Pero en cuanto a la vivienda, la situación continuó siendo infernal y la sanidad muy deficiente.

En Güira de Melena, las iniciativas autonomistas y la ayuda norteamericana en alimentos, contribuyeron a disminuir la alta tasa de mortandad de la población. En enero se registraron 121, en febrero 98, marzo 99 y abril 85. Pero la declaración de guerra de la Administración de William Mackinley a España y la imposición del bloqueo naval el 22 de abril de 1898, volvieron a elevar el número de muertes.

Los efectos del bloqueo naval norteamericano suprimió las diferencias de condiciones de vida entre reconcentrados y gran parte del resto de la población. Pues la hambruna se extendió a otros sectores sociales sin suficientes recursos monetarios para afrontar los altísimos precios que se elevaron a causa de la aguda escasez, el acaparamiento y la especulación desenfrenada.

5 ANC, Gobierno Autonómico. Leg. 17, N. ${ }^{\circ} 23$. 
Si observamos el comportamiento del número de fallecidos desde la implantación del bloqueo naval, apreciaremos una tendencia alcista que sólo se interrrumpe en julio. Y comienza a descender en los dos últimos meses del año.

$\begin{array}{lrlr}\text { Mayo } & 101 & \text { Septiembre } & 222 \\ \text { Junio } & 103 & \text { Octubre } & 133 \\ \text { Julio } & 99 & \text { Noviembre } & 87 \\ \text { Agosto } & 159 & \text { Diciembre } & 79\end{array}$

Fuente: Actas de defunciones del Registro Civil de Güira de Melena. Tomo 12.

En el cuadro presentado pudiera parecer incomprensible que en septiembre - casi dos meses después de concluida la guerra hispano-cubano-americana- se registrara la cifra de muertes más alta de la población. El hecho se explica si tenemos en cuenta que al bloqueo naval se añadieron la insuficiente capacidad productiva de las zonas de cultivo, que las autoridades militares españolas cesaron su ayuda y que el Ejército cubano comenzó a depender para su subsistencia de una parte de los alimentos y medicinas que antes se destinaban a la población.

Los efectos del hambre quedan demostrados cuando examinamos las primeras diez causas de muerte en 1898.

\begin{tabular}{|c|l|c|}
\hline LUGAR & ENFERMEDADES & FALLECIDOS \\
\hline 1 & Nutricionales y carenciales & 332 \\
2 & Enteritis y gastroenteritis & 318 \\
3 & Tuberculosis & 162 \\
4 & Paludismo & 148 \\
5 & Del corazón y vasos sanguíneos & 57 \\
6 & Cerebro vasculares & 51 \\
7 & Disentería & 29 \\
8 & Viruela & 25 \\
9 & Bronco neumonía y Neumonía & 24 \\
10 & Meningitis y Meningoencefalitis & 23 \\
\hline
\end{tabular}

Fuente: Actas de defunciones del Registro Civil de Güira de Melena. Tomo 12.

Si comparamos la ubicación de las enfermedades en 1898 con el año de 1897, observaremos cómo las nutricionales y carenciales pasaron del quinto al primer lugar con el $24,05 \%$. Seguidas de la

R. I., $1998, \mathrm{n}^{\circ} 212$ 
enteritis y gastroenteritis, con el 23,04\%. La ubicación de estas enfermedades como causa de muertes en un año en el cual la guerra había concluido cinco meses atrás demuestra que los efectos de la Reconcentración no podemos limitarlo al período bélico. Pues en 1899, aunque el número de muertes se redujo a un nivel inferior al registrado antes de la guerra, aún morían personas por haber padecido de una insuficiencia alimentaria ${ }^{6}$.

\section{IV}

Precisar las consecuencias negativas de la Reconcentración para la economía y sociedad cubana en su etapa de reconstrucción en los primeros años de República es muy difícil, por no decir imposible.

En la vertiente demográfica jamás podremos conocer el número exacto de muertes derivadas exclusivamente de la Reconcentración. Como en los pueblos y ciudades se cavaron fosas comunes, cientos de cadáveres fueron sepultados sin el registro oficial.

Por otra parte, en las zonas rurales donde habían fincas e ingenios azucareros fortificados trabajaron y vivieron reconcentrados que fallecieron y cuyos enterramientos no se informaron debido a la distancia y la situación de guerra que dificultaba el traslado a la zona urbana.

Además, a los pueblos arribaban reconcentrados en solitario de otras localidades sin la cédula de identidad y al morir no quedaba constancia.

Pero el obstáculo mayor para precisar el número de reconcentrados muertos radica en las mismas actas de defunciones y en los libros de enterramientos porque no siempre expresaba esa condición del fallecido.

\footnotetext{
6 Según las actas de defunciones de Güira de Melena, en los primeros cinco meses el comportamiento mensual fue el siguiente:

Enero 45

Febrero 22

Marzo 32

Abril $\quad 19$

Mayo 17

Esta reducción se debió a la campaña sanitaria emprendida por las autoridades norteamericanas que ocupaban la Isla.
} 
Condicionada por las dificultades expuestas, la historiografía que ha abordado el número de muertos por la Reconcentración ha tenido que recurrir a cálculos aproximados. Por eso las cifras expuestas expresan una disparidad asombrosa. Pues algunos han estimado en 500.000 las víctimas, mientras que otros la fijan entre $300.000 \mathrm{y}$ 250.000 .

No obstante, limitar la magnitud del problema demográfico a los reconcentrados nos daría una visión muy parcial de su verdadero alcance en lo económico social. Pues los efectos de la Reconcentración en cuanto a la carencia de alimentos y el empeoramiento sanitario de pueblos y ciudades, también llevó a la muerte a miles de vecinos humildes que dependían de las labores agrícolas y residían en las zonas urbanas.

Para una mejor comprensión del impacto de la Reconcentración, en la vertiente demográfica, revisemos el comportamiento de las muertes de acuerdo con los grupos de edades en Güira de Melena.

\begin{tabular}{lcccc} 
& \multicolumn{2}{c}{ AÑo 1897} & \multicolumn{2}{c}{ AÑ 1898} \\
EDAD & TOTAL & $\%$ & TOTAL & $\%$ \\
de 1 a5 & 550 & 38,40 & 341 & 24,70 \\
de 6 a 14 & 195 & 13,50 & 170 & 12,31 \\
de 15 a 49 & 393 & 27,22 & 503 & 36,48 \\
de 50 a 64 & 142 & 9,83 & 197 & 14,27 \\
de 65 y más & 164 & 11,35 & 169 & 12,24 \\
Fuente: Actas de defunciones del Registro Civil de Güira de Melena. Tomos 11 y 12.
\end{tabular}

Como podrá observarse, fueron los niños menores de cinco años los que más sufrieron los efectos de la Reconcentración, si sumamos los totales de los años de 1897 y 1898 . No obstante, es significar que históricamente la mortandad infantil en Cuba fue muy alta. Por ejemplo, en 1893, de 296 muertes registradas, 145 fueron niños menores de cinco años para un $49,48 \%$. Y en el segundo semestre de 1896 , en el cual fallecieron 182 personas, 74 correspondieron a ese grupo de edad para alcanzar el $40,65 \%$.

Durante la Reconcentración las personas de sexo masculino murieron más que las femeninas. Esto no deviene en novedad, más bien confirmó la tendencia histórica. En 1897 llegaron al 54,29\% y en 1898 al 54,21. En los por cientos expuestos no influyó de forma de-

R. I., $1998, \mathrm{n}^{\circ} 212$ 
terminante las muertes en acciones bélicas. Pues las cifras fueron extremadamente bajas, como expresamos con anterioridad.

La exposición comparativa de las muertes por razas es muy complicada, al no disponer de cifras actualizadas que precisen el número de población por razas. En el caso concreto de Güira de Melena los blancos registraron el $81,57 \%$ y el $77,02 \%$ para los años de 1897 y 1898, respectivamente. Mientras que los negros alcanzaron el 13,53\% y el $16,76 \%$. Por su parte los pardos llegaron al 4,84 y 5,57\%. Los por cientos alcanzados por los asiáticos fueron insignificantes.

Los datos expuestos incentivan la curiosidad científica, pues Güira de Melena se había caracterizado por tener una población negra y mulata relativamente alta debido a su alto número de ingenios $\mathrm{y}$ trapiches, cafetales y vegas de tabaco que le proporcionaron una elevada cifra de esclavos.

En relación al estado civil de los fallecidos, las actas de defunciones muchas veces no lo explicaban. Sin embargo, la tendencia predominante en la población adulta correspondió a los solteros, seguidos por los casados y viudos.

Una de las consecuencias más relevante de la Reconcentración fue el amplio e intenso movimiento migratorio interno. Impulsado por la sobrevivencia los campesinos reconcentrados y las personas privadas del trabajo agrícola, marcharon con sus familias a pueblos y ciudades con más potencialidades. Una de las provincias que más éxodo experimentó fue Pinar del Río, debido a su pobre desarrollo económico y a la intensidad de la guerra.

El proceso migratorio introdujo no sólo una profundización en la diferencia poblacional en la relación campo-ciudad, sino también entre las provincias y términos municipales más afectados por Reconcentración.

Este proceso migratorio no se ha tenido en cuenta para explicar las causas del aumento o disminución de la población en muchos pueblos pertenecientes al occidente de la Isla al concluir la guerra. Pues siempre se ha razonado sobre los efectos originados por la $\mathrm{Re}$ concentración. Ese es el caso de Güira de Melena, que en el censo de 1899 aparece con una población incrementada en 2.827 habitantes cuando se le compara con la que tenía en 1887. El hecho da la falsa imagen de un lugar en el cual la mortandad por la Reconcentración fue ínfima. Pero la realidad histórica, como hemos expuesto, nos dice que no fue así. 
Aunque no se dispone de fuentes con informaciones cuantificativas acerca de ese flujo migratorio, en las actas de defunciones hallamos una referencia que ilustra sobre su magnitud. En 1897 fallecieron 393 personas en Güira de Melena, que procedían de la región vueltabajera y de pueblos limítrofes. Esa cifra representa el $27,21 \%$ de las muertes de ese año. Y en 1898, con 335, alcanzaron el $24,27 \%$. Sin duda que en estos números hallamos una aproximación del volumen de la corriente migratoria interna.

Otra de las incidencias de la Reconcentración fue la saturación que experimentaron algunas ciudades y pueblos. Son notables las disposiciones del Consejo de Secretarios del gobierno autonomistas de presionar y obligar a los reconcentrados para que retornaran a sus labores agrícolas.

$\mathrm{Al}$ principio los argumentos para no regresar se basaban en el peligro que corrían a causa de la guerra. Y después era que habían hallado trabajo. Otros que habían vendido sus fincas o perdido el arrendamiento. Algunas familias alegaron que habían muerto los principales que labraban la tierra, como padres, esposos y hermanos mayores. Y, por tanto, se habían quedado sin recursos económicos. Todo esto condujo a una reestructura agraria y modificación de la composición social del campo que aún no se ha investigado acuciosamente.

Otra de las consecuencias nefastas de la Reconcentración fue la incentivación de la prostitución, la delincuencia y la corrupción de menores. Estas manifestaciones alcanzaron tal magnitud que los autonomistas elaboraron disposiciones para reprimirlas. Pero las medidas tuvieron poca efectividad porque la lucha por la sobrevivencia obligaba a las mujeres a prostituirse. Muchos niños huérfanos se convirtieron en pillos de calle y los padres de familia buscaron el sustento a cualquier precio. Esta situación de hambre y enfermedades fue aprovechada por los escrupulosos que siempre florecen en estas circunstancias 7 .

Las huellas traumáticas de la Reconcentración en la familia y sociedad cubana fueron más amplias y profundas. Sus efectos continuaron presentes hasta bien entrada la etapa republicana. Sobre todo como lesión psicológica y psíquica.

\footnotetext{
$7 \mathrm{Al}$ respecto existen referencias importantes en periódicos de la época y libros testimoniales.
}

R. I., $1998, \mathrm{n}^{\circ} 212$ 
This is a case study of the resettlement program carried out by the Spanish administration during the Cuban War of Independence, and includes a discussion of the social, economic and historical aspects of the case. By relying on primary sources, the author emphasizes the large number of deaths among civilians bought about by the program, the causes of these deaths and the catastrophic demographic trend that they reveal. 